\title{
Correspondence
}

To the Editors:

\section{Article on "Diagnosis and treatment of rheumatic fever" SLJCH 2007; 36:60-64 by Dr. G N Lucas}

\author{
Sri Lanka Journal of Child Health, 2007; 36: 121
}

Whenever I get an opportunity I browse your journal and enjoy some of the informative articles. In my opinion the above article has covered only part of the problem. The important aspect of long term prophylactic treatment is not addressed. I wonder whether Dr. Lucas is considering another article to cover that aspect.

Sincere regards,

Professor R L Jayakody

Professor of Pharmacology

\section{Response by Dr. G N Lucas}

I agree with Professor Jayakody that long term prophylactic treatment is an important aspect of rheumatic fever. I will now briefly address this aspect of rheumatic fever.

\section{Secondary prophylaxis (long-term preventive therapy in patients with a history of acute rheumatic fever or rheumatic heart disease)}

Prevention of recurrences of acute rheumatic fever is most effective by monthly injections of 1.2 million units $(600,000$ units if bodyweight $<27 \mathrm{~kg}$ ) of intramuscular benzathine penicillin $\mathrm{G}^{1}$. In some parts of the world where rheumatic fever is still quite prevalent, such as Taiwan and Brazil, a few breakthroughs of recurrences have been reported on the monthly benzathine penicillin $\mathrm{G}$ regimen. In these reports, injections of the compound every three weeks have been recommended ${ }^{2}$.

Oral prophylactic regimens are also effective but are less reliable. They are recommended when the risk of rheumatic recurrences is relatively low. Phenoxymethylpenicillin (penicillin V) is recommended in doses of $250 \mathrm{mg}$ orally twice daily. For the patient who is sensitive to penicillin, erythromycin may be substituted in a dose of $250 \mathrm{mg}^{\text {twice daily }}{ }^{3}$.

The optimal duration of antistreptococcal prophylaxis is uncertain. Children without carditis should receive prophylaxis for 5 years or up to age 18 years (whichever is longer) ${ }^{3}$. If there is mild or healed carditis duration of prophylaxis should be 10 years since the last episode or age 25 years (whichever is longer) ${ }^{3}$. If the carditis is more severe or valve surgery has been performed prophylaxis should be lifelong ${ }^{3}$. Treatment for an indefinite period is also required for those with frequent exposure to streptococci or for those who are difficult to monitor. The decision to withdraw antibacterial drugs should be individualised after carefully assessing the risk of repetitive exposures.

\section{References}

1. Dajani A, Taubert K, Ferreri P, et al, Treatment of acute streptococcal pharyngitis and prevention of rheumatic fever: A statement for health professionals. Committee on Rheumatic Fever, Endocarditis, and Kawasaki Disease of the Council on Cardiovascular Disease in the Young, the American Heart Association. Pediatrics. 1995; 96:758-64.

2. Lue HC, Wu MH, Wang JK, Wu FF, Wu YN, Three- versus four-week administration of benzathine penicillin G: effects on incidence of streptococcal infections and recurrences of rheumatic fever. Pediatrics 1996; 97:984-8.

3. WHO. Rheumatic fever and rheumatic heart disease: report of a WHO Expert Consultation, Geneva, 29 October-1 November 2001.Geneva: World Health Organization, 2004.

Dr G N Lucas

Paediatrician \& Joint Editor 